AperTO - Archivio Istituzionale Open Access dell'Università di Torino

A natural upper bound to the accuracy of predicting protein stability changes upon mutations

This is a pre print version of the following article:

Original Citation:

Availability:

This version is available http://hdl.handle.net/2318/1702387

since 2019-05-22T09:23:38Z

Published version:

DOI:10.1093/bioinformatics/bty880

Terms of use:

Open Access

Anyone can freely access the full text of works made available as "Open Access". Works made available under a Creative Commons license can be used according to the terms and conditions of said license. Use of all other works requires consent of the right holder (author or publisher) if not exempted from copyright protection by the applicable law. 


\title{
A natural upper bound to the accuracy of predicting protein stability changes upon mutations
}

\author{
Ludovica Montanucci ${ }^{1}$, Pier Luigi Martelli ${ }^{2}$, Nir Ben-Tal ${ }^{3 *}$, Piero Fariselli ${ }^{1 *}$ \\ ${ }^{1}$ Department of Comparative Biomedicine and Food Science, Legnaro (Padova), Italy. \\ ${ }^{2}$ Biocomputing Group, Department of Pharmacy and Biotechnology, University of Bologna, Bologna, Italy. \\ ${ }^{3}$ Department of Biochemistry and Molecular Biology, George S. Wise Faculty of Life Sciences, Tel Aviv University.
}

\begin{abstract}
Accurate prediction of protein stability changes upon single-site variations $(\Delta \Delta \mathrm{G})$ is important for protein design, as well as our understanding of the mechanism of genetic diseases. The performance of high-throughput computational methods to this end is evaluated mostly based on the Pearson correlation coefficient between predicted and observed data, assuming that the upper bound would be 1 (perfect correlation). However, the performance of these predictors can be limited by the distribution and noise of the experimental data. Here we estimate, for the first time, a theoretical upper-bound to the $\Delta \Delta \mathrm{G}$ prediction performances imposed by the intrinsic structure of currently available $\Delta \Delta \mathrm{G}$ data.

Given a set of measured $\Delta \Delta \mathrm{G}$ protein variations, the theoretically "best predictor" is estimated based on its similarity to another set of experimentally determined $\Delta \Delta \mathrm{G}$ values. We investigate the correlation between pairs of measured $\Delta \Delta \mathrm{G}$ variations, where one is used as a predictor for the other. We analytically derive an upper bound to the Pearson correlation as a function of the noise and distribution of the $\Delta \Delta \mathrm{G}$ data. We also evaluate the available datasets to highlight the effect of the noise in conjunction with $\Delta \Delta \mathrm{G}$ distribution. We conclude that the upper bound is a function of both uncertainty and spread of the $\Delta \Delta \mathrm{G}$ values, and that with current data the best performance should be between $0.7-0.8$, depending on the dataset used; higher Pearson correlations might be indicative of overtraining. It also follows that comparisons of predictors using different datasets are inherently misleading.
\end{abstract}

\section{Introduction}

Prediction of protein stability changes upon mutation is a crucial step in the understanding of protein function in health and disease, and may facilitate protein design. Several computational methods have been developed to predict the direction (stabilizing vs. destabilizing), and the magnitude of the perturbation of the stability of a protein introduced by a single-point mutation in its sequence (Topham et al., 1997; Zhou and Zhou 2002; Capriotti et al., 2005; Cheng et al., 2006; Capriotti et al., 2008; Parthiban et al., 2006; Yin et al., 2007; Huang et al., 2007; Masso et al., 2008; Teng et al., 2010; Worth et al., 2011; Wainreb et al., 2011; Chen et al., 2013; Giollo et al., 2014; Pires et al., 2014a; Pires et al., 2014b; Fariselli et al., 2015; Laimer et al., 2016; Folkman et al., 2016; Savojardo et al., 2016: Pucci et al., 2017). Most of these methods are based on machine learning, and, at state of the art, their Pearson correlation performances range from 0.5 to 0.8 .

The development of prediction methods has been made possible by compilations of datasets of experimentally measured changes in protein stability upon single-point variations $\left(\Delta \Delta \mathrm{G}=\Delta \mathrm{G} \_\right.$wildtype - $\Delta \mathrm{G} \_$mutant, the folding free energy difference between the wildtype and mutant protein; Guerois et al., 2002; Kumar et al., 2006; Dehouck et al., 2011; Pires et al., 2014; Broom et al., 2017). Among these, ProTherm (Kumar et al., 2006) is the most comprehensive dataset, aiming at collecting from primary literature all the experimentally determined thermodynamic values for calorimetry experiments in protein and protein mutants and making them available in a unified format. Therefore, data collected in ProTherm come from experiments performed with different techniques and in different environmental conditions. Each experimental condition is defined by several characteristics, such as $\mathrm{pH}$, temperature, additives in the solution, salt concentration, ion concentration, protein concentration, the concentration of the denaturant, addition of peptides to the protein sequence, etc. 
Different environmental conditions, such as $\mathrm{pH}$ and temperature, yield different $\Delta \Delta \mathrm{G}$ values. In Keeler et al., 2009, the stability change of variation $\mathrm{H} 180 \mathrm{~A}$ in the human prolactin $(2 \mathrm{Q} 98)$ was measured at the same temperature $\left(25^{\circ} \mathrm{C}\right)$ but in two different $\mathrm{pH}$ conditions: $\mathrm{pH}=5.8$ and $\mathrm{pH}=7.8$. The corresponding $\Delta \Delta \mathrm{G}$ values are $1.39 \mathrm{kcal} / \mathrm{mol}$ and $-0.04 \mathrm{kcal} / \mathrm{mol}$, respectively. Even when the temperature and $\mathrm{pH}$ are the same, two measures of $\Delta \Delta \mathrm{G}$ can differ, due to other experimental conditions or different techniques. This is the case for the E3R variation in protein $1 \mathrm{CSP}$, for which 6 different $\Delta \Delta \mathrm{G}$ values ranging from $1.4 \mathrm{kcal} / \mathrm{mol}$ to $2.4 \mathrm{kcal} / \mathrm{mol}$ were measured (Gribenko and Makhatadze 2007$)$ at the same temperature $\left(55^{\circ} \mathrm{C}\right.$ ) and $\mathrm{pH}$ (7.5) but at 6 different salt concentrations ranging from 0 - 1.0M NaCl. In Ferguson and Shaw (2002) the variant L3S of the calcium-binding protein S100B (1UWO) measured in two different conditions but at the same temperature $\left(25^{\circ} \mathrm{C}\right)$ and $\mathrm{pH}$ (7.2) yielded two $\Delta \Delta \mathrm{G}$ values of $1.91 \mathrm{kcal} / \mathrm{mol}$ and $-2.77 \mathrm{kcal} / \mathrm{mol}$. Here not only the value of the $\Delta \Delta \mathrm{G}$ changes, but also the sign, i.e., whether the mutation is stabilizing or destabilizing.

The broad range of experimental conditions in which $\Delta \Delta \mathrm{G}$ are measured increase the actual uncertainty associated with them in the databases. In other words, the error of a wet-lab experimental determination of a $\Delta \Delta \mathrm{G}$ in a single experiment can be quite small, roughly ranging from 0.1 to $0.5 \mathrm{kcal} / \mathrm{mol}$ (as an example it is $0.14 \mathrm{kcal} / \mathrm{mol}$ for thermal unfolding in Perl and Schmid, 2001 and as small as 0.06 and $0.09 \mathrm{kcal} / \mathrm{mol}$ in De Prat Gay et al., 1994). Nonetheless, the experimental conditions in which the $\Delta \Delta \mathrm{G}$ measures are carried out can vary substantially, introducing other sources of uncertainty associated with the measurements.

Given that all prediction methods exploit these data, an estimate of the theoretical upper bound for the prediction is crucial for the understanding and interpretation of the results. Here we approximate this upper bound by deriving an analytical expression, and by analyzing the experimental data. We show that the best performance depends on the dataset used, and would typically be significantly lower than expected.

\section{Theoretical estimation}

\subsection{Data uncertainty and representation of the data with a probabilistic model}

Given the broad range of values that the parameters of the experimental conditions can assume, the uncertainty associated with each $\Delta \Delta \mathrm{G}$ measurement for a set of single-point variations is greater than the actual experimental error of $0.1-0.5 \mathrm{kcal} / \mathrm{mol}$ (because it includes effects due to changes in experimental conditions). This uncertainty, indicated here as $\sigma$, can differ from one case to another.

A set of experiments performed on $N$ different protein variations produce $N$ observed $\Delta \Delta \mathrm{G}$ values indicated here as $\left\{x_{i}\right\}$. In the limit of the uncertainty $\sigma$ approaching 0 , the value of $x_{i}$ tends to the "real" $\Delta \Delta \mathrm{G}$ value for each variation. Our hypothesis here is that repeated observations $x_{i}$ for the same variation $i$ are distributed around the real $i$-th $\Delta \Delta \mathrm{G}$ value and has a standard deviation equal to the (unknown) uncertainty $\sigma_{i}$. We do not pose restrictions on the nature of the data distribution. We indicate with $\mu_{i}$ the real $\Delta \Delta \mathrm{G}$ value of a variation, determined in the wet-lab at arbitrary precision (for which all the conditions are enumerated and measured).

A set of $N$ experimentally observed $\Delta \Delta \mathrm{G}$ values $\left\{x_{i}\right\}$ can be generated by choosing each $x_{i}$ with probability given by its specific distribution $P_{i}\left(x_{i}\right)=P_{i}\left(x_{i} \mid \mu_{i}, \sigma_{i}\right)$ with mean given by the $i$-th real $\Delta \Delta \mathrm{G}$ value $\mu_{i}$ and with standard deviation given by the unknown uncertainty $\sigma_{i}$.

\subsection{Theoretical correlation between two observed distributions as a function of the variances}

We consider a sufficiently large number of samples to estimate the correlation between two sets of observations and hence establish a general analytical expression for the correlation between them. This is particularly useful if we want to use a set of observed $\Delta \Delta \mathrm{G}$ values $\left\{x_{i}\right\}$ as predictors for another set of observed $\Delta \Delta \mathrm{G}$ values $\left\{y_{i}\right\}$. Because we assume that no computational method can predict better than another set of experiments conducted in similar conditions, this correlation represents an upper bound for the correlation that any predictor that uses $\left\{x_{i}\right\}$ as a training set can achieve. Our goal is to estimate the correlation between a pair of sets of experimental observations, $\left\{x_{i}\right\}$ and $\left\{y_{i}\right\}$, of the same set of variations with real $\Delta \Delta \mathrm{G}$ values equal to $\left\{\mu_{i}\right\}$. We assume that both $\left\{x_{i}\right\}$ and $\left\{y_{i}\right\}$ are derived from the same set of distributions $\left(P_{i}\left(x_{i}\right)=\right.$ $\left.P_{i}\left(x_{i} \mid \mu_{i}, \sigma_{i}\right)=P_{i}\left(y_{i}\right)=P_{i}\left(y_{i} \mid \mu_{i}, \sigma_{i}\right)\right)$, where the distributions $P_{i}$ can differ for each protein variation $i$. In the following, we only assume that the means and variances are finite, with common definitions 


$$
\begin{gathered}
\mu_{i}=\left\langle y_{i}\right\rangle=\left\langle x_{i}\right\rangle=\int_{-\infty}^{\infty} x_{i} P_{i}\left(x_{i}\right) d x_{i} \\
\sigma_{i}^{2}=\left\langle\left(y_{i}-\mu_{i}\right)^{2}\right\rangle=\left\langle\left(x_{i}-\mu_{i}\right)^{2}\right\rangle=\int_{-\infty}^{\infty}\left(x_{i}-\mu_{i}\right)^{2} P_{i}\left(x_{i}\right) d x_{i}
\end{gathered}
$$

where the angular brackets represent expected values. With this notation, the expectation of the Pearson correlation is defined as:

$$
\langle\rho\rangle=\left\langle\frac{\sigma_{x y}}{\sigma_{x} \sigma_{y}}\right\rangle
$$

Considering a sufficiently large number of samples, we can set $\sigma_{x} \cong \sigma_{y}$, since they are computed in the same way from the same distributions. The Pearson denominator simplifies as $\sigma_{x} \sigma_{y} \cong \sigma_{x}{ }^{2} \cong{\sigma_{y}}^{2}$ (which is the variance of one of the two variables). To work out an analytical solution, instead of computing the expectation of the Pearson directly, we compute the ratio of the expectations of the numerator and denominators. In general, this is correct only to the first order, however, when the number of samples is sufficiently large, the Pearson correlation $\rho$ is independent of the variance $\sigma_{x}{ }^{2}$, so that the covariance between them is zero $\left(\operatorname{Cov}\left(\rho, \sigma_{x}^{2}\right) \cong 0\right)$. We can see this by generating an infinite set of different variance values by scaling the original variables $\left(x_{i}^{\prime}=k \cdot x_{i}^{\prime}\right.$ and $\left.y_{i}^{\prime}=k \cdot y_{i}\right)$, while maintaining the same Pearson value $\rho$. Since it is possible to write the covariance as a function of expectations (Heijmans, 1999) as

$$
\operatorname{Cov}\left(\rho, \sigma_{x}^{2}\right)=\left\langle\sigma_{x y}\right\rangle-\langle\rho\rangle\left\langle\sigma_{x}^{2}\right\rangle
$$

from the independence of Person and variance $\left(\operatorname{Cov}\left(\rho, \sigma_{x}^{2}\right) \cong 0\right)$ it follows that the expected value of the Pearson correlation can be approximated as

$$
\langle\rho\rangle \cong\left\langle\sigma_{x y}\right\rangle /\left\langle\sigma_{x}^{2}\right\rangle
$$

The numerator in Eq. 4 can be computed by taking the expected values of the variables $\left\{x_{i}\right\}$ and $\left\{y_{i}\right\}$, as:

$$
\left\langle\sigma_{x y}\right\rangle=\left\langle\frac{1}{N} \sum_{i}\left(x_{i}-\bar{x}\right)\left(y_{i}-\bar{y}\right)\right\rangle=\frac{1}{N} \sum_{i} \int\left(x_{i}-\bar{x}\right) P_{i}\left(x_{i}\right) d x_{i} \int\left(y_{i}-\bar{y}\right) P_{i}\left(y_{i}\right) d y_{i}
$$

where $\bar{x}$ and $\bar{y}$ are the mean values of variables $\left\{x_{i}\right\}$ and $\left\{y_{i}\right\}$. The first term in the arguments of the two integrals can be expanded by adding and subtracting the same value, in this case we choose to add and subtract the real value for the distributions: $\mu_{i}$. Hence, the argument of the first integral $\left(x_{i}-\bar{x}\right)$ can be expanded as $\left(x_{i}-\mu_{i}\right)+\left(\mu_{i}-\bar{x}\right)$. Likewise, in the second integral, $\left(y_{i}-\bar{y}\right)$ can be expanded as $\left(y_{i}-\mu_{i}\right)+\left(\mu_{i}-\bar{y}\right)$. The resulting formula becomes:

$$
\left\langle\sigma_{x y}\right\rangle=\frac{1}{N} \sum_{i}\left[\left(\int\left(x_{i}-\mu_{i}\right) P_{i}\left(x_{i}\right) d x_{i}+\int\left(\mu_{i}-\bar{x}\right) P_{i}\left(x_{i}\right) d x_{i}\right) \cdot\left(\int\left(y_{i}-\mu_{i}\right) P_{i}\left(y_{i}\right) d y_{i}+\int\left(\mu_{i}-\bar{y}\right) P_{i}\left(y_{i}\right) d y_{i}\right)\right]
$$

We notice that the first and third integrals go to zero (as $N$ increases), by definition of the mean (Eq. 1), while the second and fourth integrals, after taking out the terms that do not depend on the integration variable, give 1 (for the normalization of the distribution $P_{i}$ ). So the numerator becomes:

$$
\frac{1}{N} \sum_{i}\left(\int\left(\mu_{i}-\bar{x}\right) P_{i}\left(x_{i}\right) d x_{i}\right)\left(\int\left(\mu_{i-} \bar{y}\right) P_{i}\left(y_{i}\right) d y_{i}\right)=\frac{1}{N} \sum_{i}\left(\mu_{i}-\bar{x}\right)\left(\mu_{i}-\bar{y}\right)
$$

Assuming that the two experimental sets are derived from the same distribution, with an average of $\bar{\mu}$ (which would be the case unless there are systematic errors), $\bar{x}$ and $\bar{y}$ would equal $\bar{\mu}$, and the numerator in Eq. 4 would tend to:

$$
\sigma_{D B}^{2}=\frac{1}{N} \sum_{i}\left(\mu_{i}-\bar{\mu}\right)^{2}
$$

This is the variance of the distribution of the real $\Delta \Delta \mathrm{G}$ values, which does not depend on the experimental uncertainty but only on the distribution of the $\Delta \Delta \mathrm{G}$ values in our database (hence $\sigma_{D B}$ ). The database distribution can be of any type, the only value we consider is its variance $\sigma_{D B}{ }^{2}$. So we can estimate the covariance as:

$$
\left\langle\sigma_{x y}\right\rangle \cong \sigma_{D B}^{2}
$$


For the denominator of the Pearson correlation (Eq. 4) we have:

$$
\sigma_{x}^{2}=\frac{1}{N} \sum_{i}\left(x_{i}-\bar{x}\right)^{2}
$$

We can estimate the variance as before, thus for $\sigma_{x}^{2}$ we obtain:

$$
\begin{gathered}
\left\langle\sigma_{x}^{2}\right\rangle=\left\langle\frac{1}{N} \sum_{i}\left(x_{i}-\bar{x}\right)^{2}\right\rangle=\frac{1}{N} \sum_{i} \int\left(x_{i}-\bar{x}\right)^{2} P_{i}\left(x_{i}\right) d x_{i}= \\
=\frac{1}{N} \sum_{i} \int\left[\left(x_{i}-\mu_{i}\right)+\left(\mu_{i}-\bar{x}\right)\right]^{2} P_{i}\left(x_{i}\right) d x_{i}= \\
=\frac{1}{N} \sum_{i} \int\left[\left(x_{i}-\mu_{i}\right)^{2}+\left(\mu_{i}-\bar{x}\right)^{2}+2\left(x_{i}-\mu_{i}\right)\left(\mu_{i}-\bar{x}\right)\right] P_{i}\left(x_{i}\right) d x_{i}
\end{gathered}
$$

When integrated, the last term of the integral goes to zero as $N$ increases (based on the definition of mean, Eq. 1), while the first and the second approach the uncertainty ${\sigma_{i}}^{2}$ associated with each $\Delta \Delta \mathrm{G}$ point, and ${\sigma_{D B}}^{2}$ of the real data set, respectively. Thus, the variance can be estimated as:

$$
\left\langle\sigma_{x}^{2}\right\rangle \cong{\overline{\sigma^{2}}}^{2}{\sigma_{D B}}^{2}
$$

Where we indicate with $\overline{\sigma^{2}}$ the average variance of the data

$$
\overline{\sigma^{2}}=\frac{1}{N} \sum_{i} \sigma_{i}^{2}
$$

and with $\bar{\sigma}$ its square toot $\left(\bar{\sigma}=\sqrt{\overline{\sigma^{2}}}\right)$. With this, the Pearson correlation (Eq. 4) can be estimated as:

$$
\langle\rho\rangle \cong \frac{\left\langle\sigma_{x y}\right\rangle}{\left\langle\sigma_{x}^{2}\right\rangle} \cong \frac{\sigma_{D B}{ }^{2}}{\sigma^{2}+\sigma_{D B}{ }^{2}}=\frac{1}{1+\left(\frac{\overline{\sigma^{2}}}{\sigma_{D B}^{2}}\right)}
$$

Given that the two variances are greater than zero, the experimental observations will always yield Pearson correlation smaller than 1. The magnitude of the reduction of the correlation is imposed by the squared ratio of the average uncertainty of each data point $(\Delta \Delta \mathrm{G}$ value) and the spread of the set of data used for the prediction. Equation 14 indicates that the smaller the dispersion of the dataset ${\sigma_{D B}}^{2}$, the more sensitive the Person correlation is to the data noise (or uncertainty, $\bar{\sigma}$ ). In other words, two datasets that share the same average uncertainty but differ in their data distribution have different upper bound Pearson correlations.

Figure 1 shows a graphical plot of the correlation $\rho$ as a function of the average uncertainty $\bar{\sigma}$ and the standard deviation $\sigma_{D B}$. Each curve represents the upper bound that a Pearson correlation can achieve. For example, with $\sigma_{D B}$ of $2 \mathrm{kcal} / \mathrm{mol}$ and average uncertainty $\bar{\sigma}$ of $1 \mathrm{kcal} / \mathrm{mol}$, the maximum Pearson correlation that any predictor can achieve is only about 0.8 . 


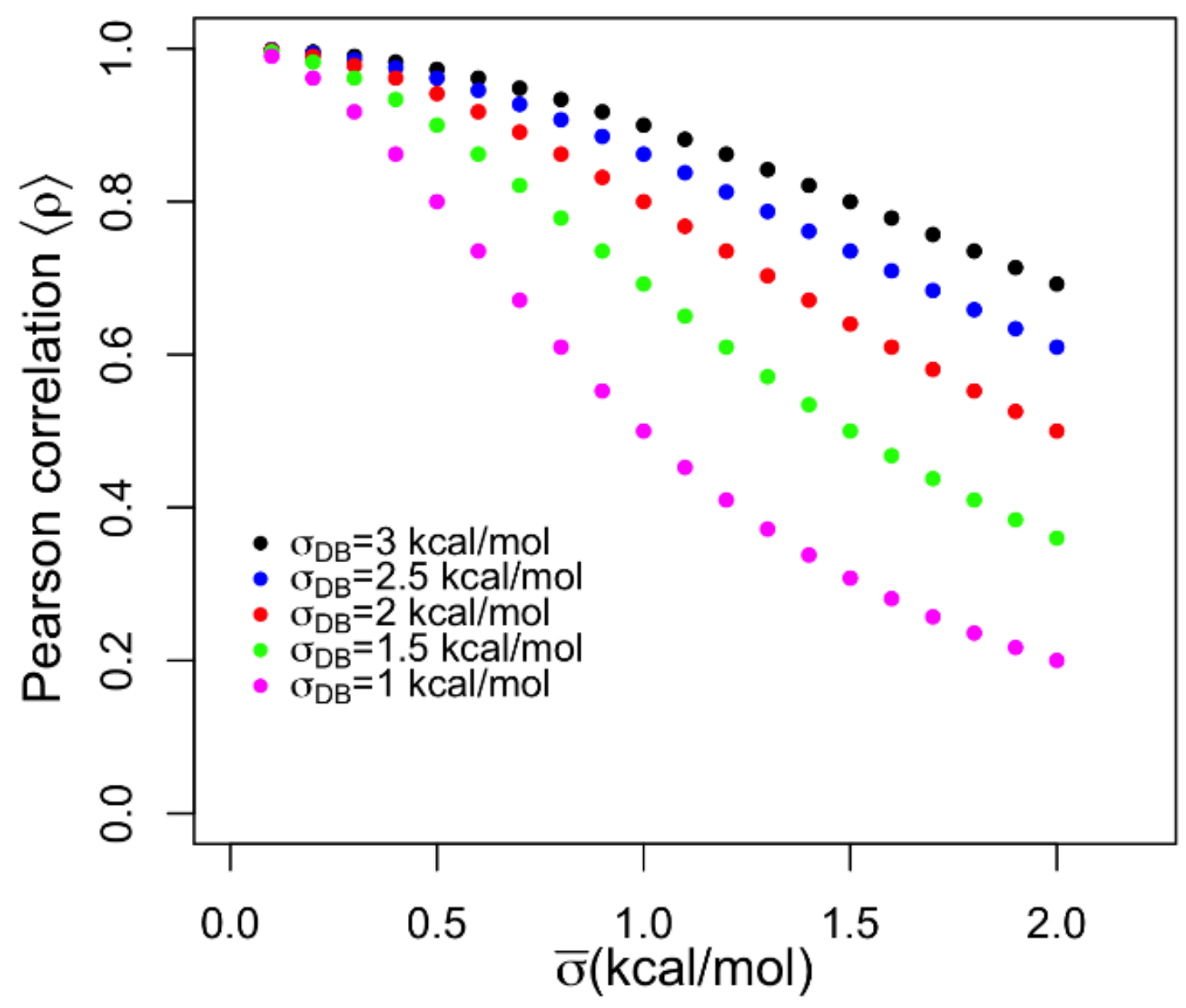

Fig. 1. Expected Pearson correlation $\left\langle\rho>\right.$ vs. data average uncertainty $(\bar{\sigma})$ for different values of dataset standard deviation $\sigma_{D B}$. The results were calculated using the approximate analytical expression of Eq. 14, with each curve corresponding to a specific $\sigma_{D B}$. The ratio between $\bar{\sigma}$ and $\sigma_{D B}$ determines the approximate upper bound of $\rho$, which, for finite values of these uncertainties, will always be smaller than 1. 


\section{Experimental datasets}

To be more realistic, we supplement the approximate theoretical derivation of section 2 with estimates based on real mutation data taken from the available databases.

\subsection{Distribution of the available data}

We considered three datasets used to train most of the available computational methods. The first is the latest version of ProTherm (Kumar et al., 2006, last update in 2013) comprising 3464 single-point mutations from 135 proteins of known structure (with PDB IDs). The second is S2648 (Dehouck et al., 2011) which comprises 2648 single-site variations in 131 proteins taken and cleaned from a previous release of ProTherm. The third is VariBench, a manually curated dataset, for which the $\Delta \Delta \mathrm{G}$ measurements have been checked in the primary literature. VariBench (Yang et al., 2018) is derived from the ProTherm release of February 22, 2013 and contains 1564 single-site variations from 99 proteins.

The three datasets have a very sharp distribution around the average value (around $-1.06 \mathrm{kcal} / \mathrm{mol}$ for each set) is observed, with low standard deviations $\left(\sigma_{D B}\right)$ of $2.06 \mathrm{kcal} / \mathrm{mol}$ in ProTherm, $1.91 \mathrm{kcal} / \mathrm{mol}$ in VariBench, and $1.47 \mathrm{kcal} / \mathrm{mol} \mathrm{in} \mathrm{S} 2648$ (Figure 1S, supplementary materials). The theoretical model above (Eq. 14) shows that with such low deviations, the Pearson correlation should be sensitive to noise.

It is also noteworthy that because of the difference in their standard deviations, the maximum possible Pearson correlation of the three datasets, for the same average experimental uncertainty $\bar{\sigma}$, is not the same. In particular, if both the VariBench and S2648 datasets are affected by the same average experimental uncertainty $\bar{\sigma}$, the maximum possible Pearson correlation of VariBench would be larger.

\subsection{What can we expect from the current experimental datasets?}

We made a raw evaluation of what we can expect from the current experimental dataset. In particular, we considered two datasets:

1) $\mathrm{S} 1$ : a subset of 574 ProTherm single-site variations for which two or more experimental $\Delta \Delta \mathrm{G}$ values are reported for the same protein variation, measured at the same temperature and $\mathrm{pH}$;

2) S2: a subset of 551 variations shared by VariBench and S2648, for which the manual curators ended up with different $\Delta \Delta \mathrm{G}$ values.

Thus, for each variation $i$ (in either S1 and S2), we can associate a mean value $\left(\mu_{i}=\overline{\Delta \Delta G}_{i}\right)$ and a standard deviation $\left(\sigma_{i}\right)$, since we have at least two $\Delta \Delta \mathrm{G}$ values. Computing $\bar{\sigma}$ (as the square root of the mean of $\left\{\sigma_{i}^{2}\right\}$ ) and the $\sigma_{D B}$ (using the $\left.\overline{\{\Delta \Delta G}_{i}\right\}$ ) for $\mathrm{S} 1$ and $\mathrm{S} 2$, we obtained $\bar{\sigma}=1.04$ and $\sigma_{D B}=1.72$, and $\bar{\sigma}=0.72$ and $\sigma_{D B}=1.57$, respectively. Substituting these values in Eq 14, we obtain estimations of maximum Pearson correlations of 0.73 for S1 and 0.83 for S2. However, the same data can be used to explicitly take into account the different $\left\{\sigma_{i}^{2}\right\}$ values. For each variation it is possible to derive a set of pairs of "experiments" by randomly drawing two values at a time from the normal distribution $N\left(\overline{\Delta \Delta G}_{i}, \sigma_{i}\right)$. Figure 2 shows the results of a typical run. By drawing 100 pairs of such "experiments", and repeating the runs 10 times, we obtained an estimation of the Pearson correlation (with variable $\left\{\sigma_{i}\right\}$ ) of $0.74 \pm 0.02$ and $0.84 \pm 0.02$, for S1 and S2 respectively. 


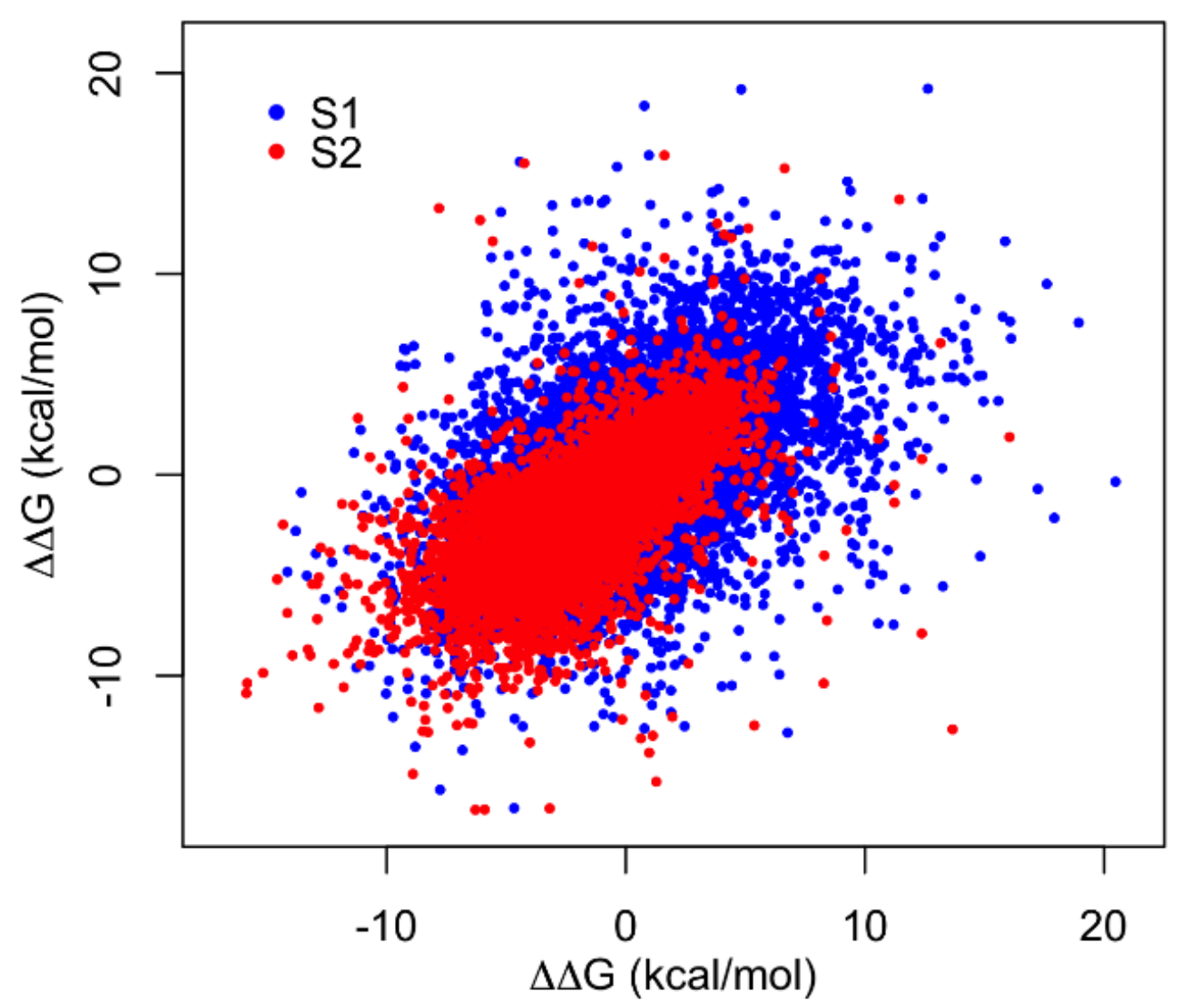

Fig. 2. Scatterplot of two randomly generated observations for a given variation. Red points are 100 randomly generated observations according to a normal distribution, with $\overline{\Delta \Delta G}_{i}$ and $\sigma_{i}$ taken from the manually curated S2: different value for the same mutation reported in S2648 and VeriBench). Blue points are 100 randomly generated observations according to a normal distribution with $\overline{\Delta \Delta G}_{i}$ and $\sigma_{i}$ taken from S1: ProTherm variations with more than one $\Delta \Delta \mathrm{G}$ value reported for the same variation at the same $\mathrm{pH}$ and Temperature.

\section{Conclusion}

Using a general model we approximated the correlation between a pair of observed $\Delta \Delta \mathrm{G}$ measurements, where one is used to predict the other. An approximate analytical expression, as well as simulations using real data, show that the correlation is limited by the ratio between these two uncertainties, placing a natural upper bound on the maximum possible Pearson correlation between predicted and empirical values. With current accuracy, the theoretical value critically depends on both the average uncertainty of the data $(\bar{\sigma})$ and the spread of the dataset used $\left(\sigma_{D B}\right)$. While the first can be reduced to some extent by manually cleaning the data, $\sigma_{D B}$ is an intrinsic property of the dataset that provides an upper bound to the maximum expected Person correlation.

A similar approach can be used to derive a lower bound for the root mean square error (Rmse), another commonly used measure of performance. In Supplementary Material we show that he expected value of the root mean square error is a linear function of the average data uncertainty $(\langle R m s e\rangle \simeq \sqrt{2} \bar{\sigma})$. The current datasets (S2648, VariBench) have a $\sigma_{D B}<2$, dictating an upper bound of about 0.8 to the Pearson correlation and lower bound of about $1 \mathrm{kcal} / \mathrm{mol}$ for the root mean square error (see Supplementary Materials); better values would be indicative of overtraining.

Generally speaking, the conclusions should be valid whenever large empirical datasets compiled of various measurements are used for training a predictor, and equation 14 gives an approximate upper bound for the prediction accuracy. 


\section{References}

Broom,A., Jacobi,Z., Trainor,K. and Meiering,E.M. (2017) Computational tools help improve protein stability but with a solubility tradeoff. J Biol Chem, 292,14349-14361.

Capriotti E, Fariselli P, Casadio R. (2005) I-Mutant2.0: predicting stability changes upon mutation from the protein sequence or structure. Nucleic Acids Res., 33, 306-310.

Capriotti,E., Fariselli,P., Rossi,I., Casadio,R. (2008) A three-state prediction of single point mutations on protein stability changes. BMC Bioinformatics., 9, S6.

Chen,C.W., Lin,J., Chu,Y.W. (2013) iStable: off-the-shelf predictor integration for predicting protein stability changes. $B M C$ Bioinformatics., 14, S5.

Cheng,J., Randall,A., Baldi,P. (2006) Prediction of protein stability changes for single-site mutations using support vector machines. Proteins, 62, 1125-1132.

De Prat Gay,G., Johnson,C.M., Fersht,A.R. (1994) Contribution of a proline residue and a salt bridge to the stability of a type I reverse turn in chymotrypsin inhibitor-2. Protein Eng., 7, 103-108.

Dehouck,Y., Kwasigroch,J.M., Gilis,D., Rooman,M. (2011) PoPMuSiC 2.1: a web server for the estimation of protein stability changes upon mutation and sequence optimality. BMC Bioinformatics, 12, 151.

Fariselli,P., Martelli,P.L., Savojardo,C., Casadio,R. (2015) INPS: predicting the impact of non-synonymous variations on protein stability from sequence. Bioinformatics, 31, 2816-2821.

Ferguson,P.L., Shaw,G.,S. (2002) Role of the N-terminal helix I for dimerization and stability of the calcium-binding protein S100B. Biochemistry, 41, 3637-3646.

Folkman,L., Stantic,B., Sattar,A., Zhou,Y. (2016) EASE-MM: Sequence-Based Prediction of Mutation-Induced Stability Changes with Feature-Based Multiple Models. J Mol Biol., 428, 1394-1405.

Giollo,M., Martin,A.J., Walsh,I., Ferrari,C., Tosatto,S.C. (2014) NeEMO: a method using residue interaction networks to improve prediction of protein stability upon mutation. BMC Genomics, 15, S7.

Gribenko,A.V., Makhatadze,G.I. (2007) Role of the charge-charge interactions in defining stability and halophilicity of the CspB proteins. J Mol Biol., 366, 842-856.

Guerois,R., Nielsen,J.E., Serrano,L. (2002) Predicting changes in the stability of proteins and protein complexes: a study of more than 1000 mutations. J Mol Biol., 320, 369-387.

Heijmans, R. (1999) When does the expectation of a ratio equal the ratio of expectations? Statistical Papers. 40, $107-115$.

Huang,L.T., Gromiha,M.M., Ho,S.Y. (2007) iPTREE-STAB: interpretable decision tree basedmethod for predicting protein stability changes upon mutations. Bioinformatics. 23, 1292-1293.

Keeler,C., Tettamanzi,M.C., Meshack,S., Hodsdon,M.E. (2009) Contribution of individual histidines to the global stability of human prolactin. Protein Sci., 18, 909-920.

Kepp,K.P. (2015) Towards a "Golden Standard" for computing globin stability: Stability and structure sensitivity of myoglobin mutants. Biochim Biophys Acta, 1854 (10 Pt A), 1239-1248.

Kumar,M.D., Bava K.A., Gromiha M.M., Prabakaran P., Kitajima K., Uedaira H., Sarai A. (2006) ProTherm and ProNIT: thermodynamic databases for proteins and protein-nucleic acid interactions. Nucleic Acids Res. (Database issue), 34, D204206.

Laimer,J., Hiebl-Flach,J., Lengauer,D., Lackner,P. (2016) MAESTROweb: a web server for structure-based protein stability prediction. Bioinformatics, 32, 1414-1416.

Masso,M., Vaisman,,I. (2008) Accurate prediction of stability changes in protein mutants by combining machine learning with structure based computational mutagenesis. Bioinformatics, 24, 2002-2009.

Parthiban,V., Gromiha,M.M., Schomburg,D.. (2006) CUPSAT: prediction of protein stability upon point mutations. Nucleic Acids Res., 34, 239-242.

Perl,D., Schmid,F.X. (2001) Electrostatic stabilization of a thermophilic cold shock protein. J Mol Biol., 313(2), $343-357$.

Pires,D.E., Ascher,D.B., Blundell,T.L. (2014a) mCSM: predicting the effects of mutations in proteins using graph-based signatures. Bioinformatics, 30, 335-342.

Pires,D.E., Ascher,D.B., Blundell,T.L. (2014b) DUET: a server for predicting effects of mutations on protein stability using an integrated computational approach. Nucleic Acids Res., 42, 314-319. 


\section{Supplementary material for the paper "A natural upper bound to the accuracy of predicting protein stability changes upon mutations"}

\section{Distribution of $\Delta \Delta \mathbf{G}$ in different datasets}

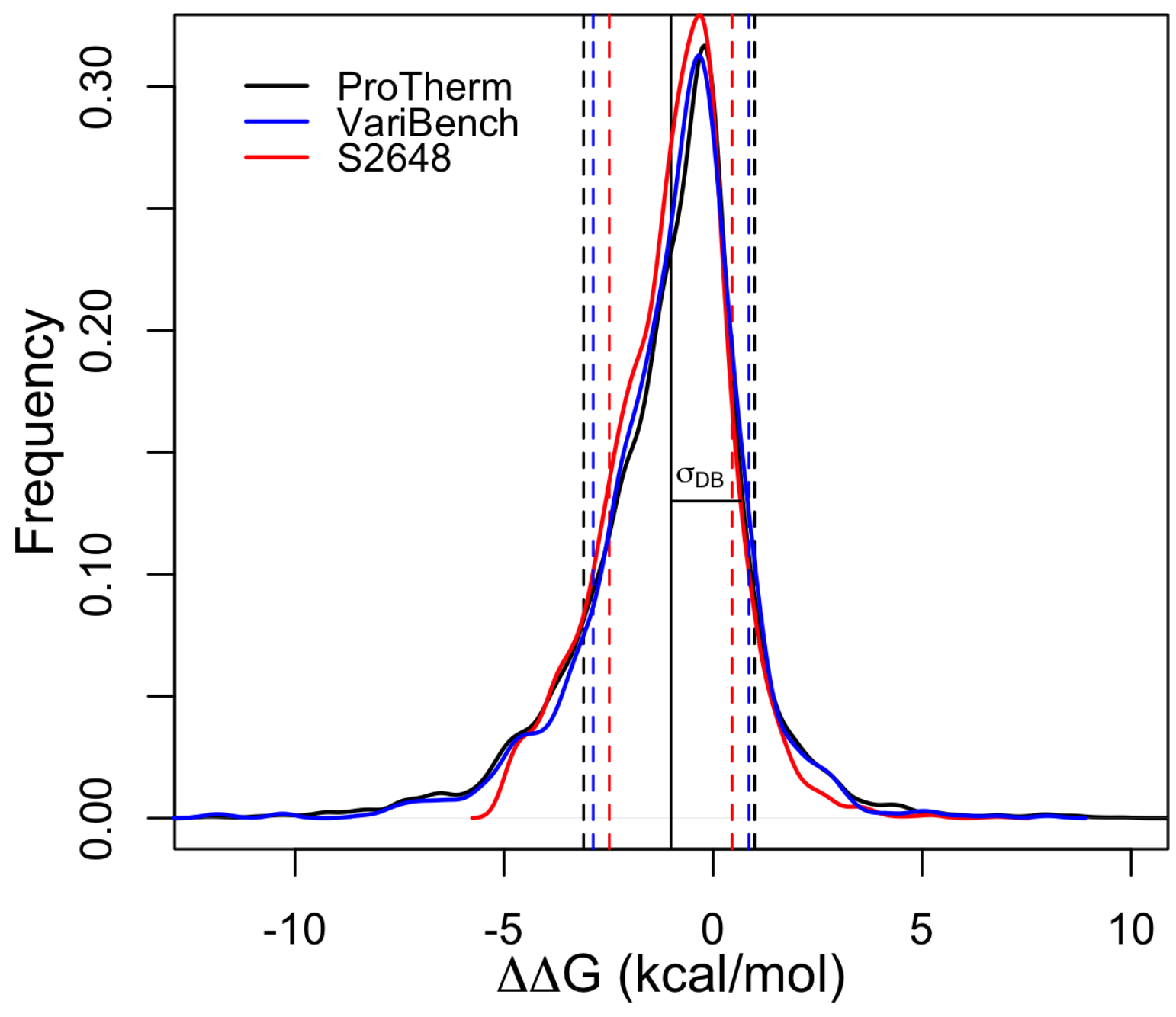

Fig. 1S. Distribution of $\Delta \Delta \mathbf{G}$ in different datasets. ProTherm in black, S2648 in blue and Varibench in red. The black solid line marks the mean, about $-1 \mathrm{kcal} / \mathrm{mol}$ for each of the three datasets. The vertical dashed lines indicate the limits of the mean \pm standard deviations of the three distributions, that are: 2.06 $\mathrm{kcal} / \mathrm{mol}$ in ProTherm (which is shown in the graph), $1.91 \mathrm{kcal} / \mathrm{mol}$ in VariBench, and $1.47 \mathrm{kcal} / \mathrm{mol}$ in S2648. 


\section{Estimation of a lower bound for the root mean square error}

A second measure of accuracy usually adopted to score predictors is the root means square error (Rmse), which is the square root of the mean square error (mse). Using a similar approach applied for the Pearson correlation, we can estimate the expected value of mse using a set of experiments $\left\{x_{i}\right\}$ as a predictor of another set of experiments $\left\{y_{i}\right\}$. In this case the expectation is

$$
\langle m s e\rangle=\left\langle\frac{1}{N} \sum_{i}\left(x_{i}-y_{i}\right)^{2}\right\rangle=\frac{1}{N} \sum_{i}\left\langle\left(x_{i}-\mu_{i}+\mu-y_{i}\right)^{2}\right\rangle
$$

by taking the squares in the round brackets, and computing the expectation using the data distributions $P_{i}\left(x_{i} \mid \mu_{i}, \sigma_{i}\right)=P_{i}\left(y_{i} \mid \mu_{i}, \sigma_{i}\right)$, and considering the independence of the variables $x$ and $y$, Eq. 15 reads as

$$
\begin{gathered}
\langle m s e\rangle= \\
\frac{1}{N} \sum_{i}\left(\left\langle\left(x_{i}-\mu_{i}\right)^{2}\right\rangle+\left\langle\left(y_{i}-\mu_{i}\right)^{2}\right\rangle-2\left\langle x_{i}-\mu_{i}\right\rangle\left\langle y_{i}-\mu_{i}\right\rangle\right)
\end{gathered}
$$

The first two terms are the distribution variances, and it follows from the definition of the mean and variance (Eq. 1) that the last term goes to zero as $\mathrm{N}$ increases. Thus we have

$$
\langle m s e\rangle=\frac{1}{N} \sum_{i}\left(2 \sigma_{i}^{2}\right)=2 \overline{\sigma^{2}}
$$

Where $\overline{\sigma^{2}}$ is the average variance of the data

$$
\overline{\sigma^{2}}=\frac{1}{N} \sum_{i} \sigma_{i}^{2}
$$

Eq.3S indicates that the mean square error is a function of the average data uncertainty and does not depend on the data distribution, so we may assume the Rmse is a linear function of the average data uncertainty, such as

$$
\langle R m s e\rangle \cong \sqrt{2} \bar{\sigma}
$$

where $\bar{\sigma}$ is the square root of the average variance $\left(\bar{\sigma}=\sqrt{\overline{\sigma^{2}}}\right)$. 
This means that the lower bound to the Rmse is provided by the average of the data uncertainty and, unlike the Pearson correlation (main text Eq. 14), does not depend on the database distribution.

\section{Experimental estimation of the root mean square error}

Considering the two sets introduced in the main text, such as:

1) S1: a subset of 574 ProTherm single-site variations for which two or more experimental $\Delta \Delta \mathrm{G}$ values are reported for the same protein variation, measured at the same temperature and $\mathrm{pH}$. S1 data uncertainty is $\bar{\sigma}=1.04$.

2) S2: a subset of 551 variations shared by VariBench and S2648, for which the manual curators ended up with different $\Delta \Delta \mathrm{G}$ values. $\mathrm{S} 1$ data uncertainty is $\bar{\sigma}=0.72$

We can estimate the $\langle R m s e\rangle$ using E. 5S for the two dataset obtain $\langle R m s e\rangle=1.46$ $\mathrm{kcal} / \mathrm{mol}$ and $\langle R m s e\rangle=1.0 \mathrm{kcal} / \mathrm{mol}$ for $\mathrm{S} 1$ and $\mathrm{S} 2$ respectively. These values agree with those obtained by extensive simulations using the procedure described in the main text (section 3.2), where for the computed values we obtain $\langle R m s e\rangle=1.4 \pm 0.5 \mathrm{kcal} / \mathrm{mol}$ and $\langle R m s e\rangle=1.0 \pm 0.2 \mathrm{kcal} / \mathrm{mol}$, for $\mathrm{S} 1$ and $\mathrm{S} 2$ respectively.

These results are perfectly in line with those obtained by our equation bound. 